\title{
émulations
}

\section{La santé face aux inégalités et aux discriminations}

\author{
Louis Braverman et Aurore Loretti
}

Émulations - Revue de sciences sociales, 2020, n 35-36, "Santé, inégalités et discriminations ».

\section{Article disponible à l'adresse suivante}

https://ojs.uclouvain.be/index.php/emulations/article/view/bravermanloretti

\section{Pour citer cet article}

Louis Braverman et Aurore Loretti, « La santé face aux inégalités et aux discriminations », Émulations, n 35-36, Mise en ligne le 30 décembre 2020.

DOI : 10.14428/emulations.03536.01

Distribution électronique : Université catholique de Louvain (Belgique) : ojs.uclouvain.be

(C) Cet article est mis à disposition selon les termes de la Licence Creative Commons Attribution, Pas d'Utilisation Commerciale 4.0 International. http://creativecommons.org/licenses/by-nc/4.0/

Éditeur : Émulations - Revue de sciences sociales / Presses universitaires de Louvain https://ojs.uclouvain.be/index.php/emulations

ISSN électronique : 1784-5734 


\title{
émulations
}

\section{La santé face aux inégalités et aux discriminations}

\author{
Louis Braverman et Aurore Loretti
}

Émulations - Revue de sciences sociales, 2020, n 35-36, "Santé, inégalités et discriminations ».

\section{Article disponible à l'adresse suivante}

https://ojs.uclouvain.be/index.php/emulations/article/view/bravermanloretti

\section{Pour citer cet article}

Louis Braverman et Aurore Loretti, « La santé face aux inégalités et aux discriminations », Émulations, n 35-36, Mise en ligne le 30 décembre 2020.

DOI : 10.14428/emulations.03536.01

Distribution électronique : Université catholique de Louvain (Belgique) : ojs.uclouvain.be

(C) Cet article est mis à disposition selon les termes de la Licence Creative Commons Attribution, Pas d'Utilisation Commerciale 4.0 International. http://creativecommons.org/licenses/by-nc/4.0/

Éditeur : Émulations - Revue de sciences sociales / Presses universitaires de Louvain https://ojs.uclouvain.be/index.php/emulations

ISSN électronique : 1784-5734 


\title{
La santé face aux inégalités et aux discriminations
}

\author{
Louis Braverman' et Aurore Loretti
}

L'étude de la différenciation sociale et celle de la (re)production des inégalités qui lui est consubstantielle constituent sans aucun doute l'un des axes centraux autour desquels s'est construite la réflexion sociologique (Dubet, 2011 ; Duvoux, 2017). Certains vont d'ailleurs jusqu'à affirmer qu'il n'y aurait de sociologie que dans l'étude « des rapports inégaux et des figures de la différence » (Passeron, 1981 : 7). Comme le pointe Bernard Lahire, « [l]es faits d'inégalité ou de domination sont têtus : ils se mesurent, s'objectivent et se constatent dans tous les domaines de pratiques » (2019: 43). Dès lors, la sociologie met inévitablement au jour les dissymétries et les structures inégalitaires qui traversent nos sociétés et s'observent dans des domaines et à des échelles très variables (Ibid. : 48).

Parce qu'elle révèle de manière tout à fait singulière « les tensions du monde social » (Scodellaro, Pan Ké Shon, Legleye, 2017: 8), la santé constitue un objet particulièrement heuristique pour traiter de ces questions. Dans ce domaine, la différenciation sociale est souvent objectivée à travers l'utilisation d'indicateurs statistiques. Parmi eux, l'espérance de vie - soit le nombre moyen d'années vécues par les membres d'une même génération - est un outil synthétique permettant de mesurer les écarts de santé entre pays ou régions, selon les catégories socioprofessionnelles, ainsi qu'entre hommes et femmes. Dans l'ensemble des pays européens, on observe que l'espérance de vie moyenne est toujours plus élevée dans les populations situées en haut de l'échelle sociale (Menvielle et al., 2009). Les écarts relevés sont substantiels avec une différence qui se situe, le plus souvent, entre 5 et 10 ans en matière d'espérance de vie à la naissance et entre 10 et 20 ans si l'on considère l'espérance de vie sans incapacité (Mackenbach et al., 2008 ; Marmot et al., 2008). En Belgique, l'espérance de vie d'un diplômé du supérieur dépasse ainsi de neuf années celle d'un diplômé de l'enseignement primaire et, en France, un homme cadre peut espérer vivre sept années de plus qu'un ouvrier (Van Oyen et al., 2011). Ces écarts sont cependant variables en fonction du sexe et ils sont moins marqués chez les femmes. En France, l'écart entre une cadre et une ouvrière n'est en effet « que » de trois années et une femme ouvrière peut même espérer vivre une année de plus qu'un homme cadre (DREES, 2017).

Paradoxalement, bien qu'elles connaissent des situations socioéconomiques plus dégradées que les hommes, les femmes vivent en moyenne plus longtemps et béné-

\footnotetext{
${ }^{1}$ Postdoctorant, Université Sorbonne Paris Nord et Institut de recherche interdisciplinaire sur les enjeux sociaux (IRIS).

2 Docteure en sociologie et maîtresse de conférences dans le laboratoire ETHICS (ea 7446) et le Centre d'éthique médicale (CEM) de l'Université catholique de Lille.
} 
ficient d'un taux de mortalité à chaque âge plus faible (DREES, 2017). Cet « avantage » féminin doit cependant être nuancé pour plusieurs raisons. Tout d'abord, les écarts observés entre les hommes et les femmes en matière de santé sont en voie de réduction. Ensuite, cette tendance n'est « pas universelle » (Hunt, Macintyre, 2000 : 373) et l'effet du sexe sur la santé peut « s'exprimer de façon différentielle selon les maladies considérées » (Gelly, 2016 : 148). Les femmes sont, par exemple, particulièrement exposées aux maladies cardiovasculaires pour lesquelles elles sont, par ailleurs, moins bien prises en charge que les hommes (Vidal, Salle, 2017). Tous ces éléments montrent à quel point les inégalités sociales de santé restent importantes, complexes et s'inscrivent dans des configurations parfois étonnantes, comparativement à d'autres domaines de la vie sociale.

\section{Quand une pandémie rencontre les inégalités}

La récente pandémie de Covid-19 nous rappelle avec force le poids des inégalités puisque ce sont les populations les plus vulnérables qui, une fois encore, sont les plus touchées. Il a été montré que nous n'avons pas tous été exposés de la même manière au risque de contamination, à celui de développer des formes graves et de mourir, ou encore aux conséquences du confinement et des mesures de distanciation physique (Arber, Meadows, 2020 ; Bajos et al. 2020 ; Bessière et al. 2020 ; Carde, 2020 ; Dubost, Pollak, Rey, 2020 ; Marmot, 2020 ; Noûs, 2020). Certains travailleurs ont été bien plus exposés que d'autres : si les deux tiers des cadres ont pu passer au télétravail, la quasi-totalité des ouvriers et les trois quarts des employés ont été contraints de continuer à travailler en dehors de chez eux (Lambert et al., 2020). Dès lors, les premières études menées indiquent que l'excédent de mortalité a été très variable selon les caractéristiques sociales des individus : ce sont en effet les plus pauvres qui ont payé le plus lourd tribut en France (en Seine-Saint-Denis, notamment), en Angleterre, en Suède ou encore aux États-Unis (Brun, Simon, 2020 ; Counil, Khlat, 2020 ; Noppert, 2020).

À la précarité s'ajoute également l'origine ethnique comme facteur étroitement associé au risque de décès (Dubost, Pollak, Rey, 2020 ; Williamson et al., 2020). En Grande-Bretagne et aux États-Unis, une mortalité plus élevée au sein de la population noire est ainsi clairement démontrée. De même, en avril 2020, la hausse des décès a été deux fois plus importante pour les personnes nées à l'étranger que pour celles nées en France (Dubost, Pollak, Rey, 2020 ; Papon, Robert-Bobée, 2020).

Les personnes âgées figurent parmi les premières victimes de la pandémie. Elles ont par conséquent été étiquetées comme les plus vulnérables, celles qu'il faut prioritairement protéger, quitte à envisager d'adopter des mesures de confinement spécifique (Balard, Corvol, 2020). Le « risque d'une instrumentalisation de la vulnérabilité » (Renard, Rebourg, 2020), c'est-à-dire la disqualification généralisée de l'autonomie d'une catégorie de personnes en raison du principe de protection ou de sécurité, plane d'ailleurs toujours sur les aîné·e·s. 
Les inégalités de genre ont également été particulièrement saillantes puisque ce sont majoritairement des femmes qui ont œuvré dans les métiers de «première ligne » (70 \% du personnel soignant est féminin dans les pays de l'OCDE), tout en ayant à supporter un surplus de travail domestique (Recchi et al., 2020) et en étant davantage exposées aux violences conjugales (Dubost, Pollak, Rey, 2020). «Sous la pandémie, le patriarcat », met ainsi en garde la revue Contretemps (2020).

Enfin, les réflexions sur les enjeux sociaux de la pandémie montrent un renforcement, voire même l'émergence de nouvelles dynamiques de discriminations, de stigmatisations et d'altérisations (Chung, Li, 2020 ; Devakumar et al., 2020). Ces dynamiques rejoignent les processus d'ethnicisation et de racisation déjà bien ancrés dans nos sociétés (Poiret, 2011). Elles vont des positions anti-migrants prises par certains gouvernements, aux rumeurs sur un complot chinois visant à déstabiliser les ÉtatsUnis, en passant par la stigmatisation des personnes d'origine asiatique (Scambler, 2020). Pour autant, la Covid-19 a également généré d'importants élans de solidarité ainsi qu'une condamnation du racisme et de la xénophobie, comme en témoigne le succès du hashtag \#JeNeSuisPasUnVirus en France.

Cette crise illustre ainsi particulièrement bien à quel point les inégalités sociales sont systémiques (Bihr, Pfefferkorn, 2008), de quelle manière elles interfèrent, se déterminent et se génèrent réciproquement tant et si bien que certains accumulent les avantages, tandis que d'autres cumulent les handicaps. Avantages et handicaps sont incorporés, façonnant des corps plus ou moins exposés aux altérations et aux diverses affections, plus ou moins résistants aux effets du temps. Si ce double numéro d'Émulations a été pensé avant la survenue de la pandémie et que les articles qui le composent n'y font pas référence, il nous semblait donc difficile de ne pas ouvrir ce dossier en mentionnant cette actualité, tant elle résonne avec les objets ici abordés et les réflexions proposées.

\section{Qualifier les inégalités et les discriminations en santé}

Cela fait maintenant une vingtaine d'années que la question des inégalités sociales de santé fait l'objet d'un regain d'intérêt dans le champ académique francophone. Après avoir été longtemps éclipsées, les recherches et les publications sur cette question se font de plus en plus nombreuses et ce numéro s'inscrit dans cette dynamique (Aïach, Niewiadomski, 2008 ; Gelly, Pitti, 2016 ; Haschar-Noé, Lang, 2018 ; HCSP, 2009 ; Lang, 2018 ; Leclerc et al., 2000). Si le constat des inégalités sociales de santé est aujourd'hui bel et bien dressé, leur compréhension reste cependant en chantier. Les études menées ont pu montrer « la multiplicité des facteurs impliqués dans la construction des inégalités, leur effet cumulatif agissant tout au long de la vie, souvent renforcé plus que réduit par le système de soins et de prévention » (Haschar-Noé, Lang, 2018 : 16). Des recherches restent donc nécessaires pour mieux comprendre les logiques complexes et enchâssées qui les sous-tendent. 
Les inégalités observées dans le domaine de la santé sont l'une des manifestations les plus saillantes de «l'inscription des structures sociales dans les corps » (Bourdieu, 1997: 206). Bien que le corps soit souvent apparu comme le lieu de la différence biologique, s'il est un endroit où se manifeste tout particulièrement le cumul des inégalités, c'est bien dans notre corporéité. Comme le décrit si justement Philippe Perrot, «l'indigence, le labeur, les maternités, la maladie marquent, usent, tordent les corps, les plient, les voûtent, les rident précocement, là où l'aisance, l'oisiveté et la santé permettent de les entretenir, de les conserver plus frais, plus lisses, et plus droits. Se dépose et s'imprime ainsi dans les chairs - et jusqu'aux os - le texte de leur histoire : les stigmates de leurs origines, les empreintes de leur trajectoire, voire les indices de leur destinée » (Perrot, 1991 : 9.). Aussi s'agit-il d'essayer de comprendre de quelle manière et par quels processus le social s'incorpore (Fassin, $2000: 14$ ) ou, pour le dire autrement, comment la culture devient nature (Bourdieu, 1979).

Dans la lignée des travaux de Didier Fassin (2000) appelant à « qualifier les inégalités ", les textes ici réunis s'appuient sur des méthodes d'enquête qualitatives afin d'appréhender les processus de constitution des inégalités. Cette perspective est assez originale puisque pendant bien longtemps les faits d'inégalités en santé ont été principalement « pensés dans le langage des chiffres » et la majorité des études menées était de nature statistique (Fassin, $2000: 123$ ). Cette prééminence des approches chiffrées est pour partie historique (Kivits et al., 2016 : 13-14) et n'est pas spécifique aux travaux francophones puisqu'un constat similaire a été fait dans la littérature anglo-saxonne (Cockerham, 2016 ; Mackenbach, Bakker, 2002). Or, si la mesure des inégalités est indispensable, en ce qu'elle permet notamment de les révéler, les qualifier, les raconter, les montrer par le biais de données qualitatives n'en est pas moins essentiel et s'inscrit dans un rapport de complémentarité. Cela permet d'approfondir l'étude de certaines dynamiques sociales à l'œuvre dans les mondes de la santé qu'une approche chiffrée ne peut tout simplement pas mettre au jour. En faisant la part belle aux approches empiriques et ethnographiques des faits d'inégalités et de discriminations, ce numéro contribue à révéler le jeu des mécanismes, formels et informels, structurels et organisationnels, ainsi que les interactions, la pluralité d'acteurs et les différents espaces qui interviennent dans la fabrique des inégalités.

Privilégier les approches qualitatives permet en outre de nourrir la discussion sur la complémentarité des méthodes et des grilles d'analyse en santé (Kivits et al., 2016). Entretiens, observations et documents d'archives sont tour à tour mobilisés pour étudier les inégalités. Si les disciplines et les paradigmes représentés dans ce dossier sont pluriels, les auteur.e.s partagent une posture compréhensive qui s'attache à traduire le sens que donnent les individus à leurs pratiques ou aux discours. Cela permet de rompre avec le caractère parfois désincarné des études statistiques sur les inégalités sociales de santé, de discerner ce qui se loge dans les expériences du quotidien et d'affiner la compréhension des rapports sociaux. 
L'accueil et le tri des patients aux urgences, le diabète, le VIH/sida, le suivi de grossesse et les parcours de contraception sont autant d'objets à partir desquels ce numéro s'intéresse au processus de constitution des inégalités de santé. Ces thèmes révèlent, au-delà de leur diversité, des enjeux et des problèmes communs : l'accès aux soins, le poids des catégorisations, l'encastrement des inégalités dans des logiques organisationnelles ou encore le cadrage par les politiques publiques sont interrogés de manière transversale.

Ce numéro couvre par ailleurs une pluralité de contextes puisque les études exposées ont été menées en France, en Suisse, en Belgique ainsi qu'au Canada. Bien que les systèmes de santé de ces pays soient classés parmi les plus «performants » au monde par l'OMS, ces recherches montrent que cela ne saurait faire oublier les inégalités qui les traversent. En contrepartie, ce numéro reste cependant centré sur les faits d'inégalités dans les pays du Nord. Cela ne doit pas éclipser ceux des Suds, où ces questions méritent également d'être investiguées et qui ont d'ailleurs fait l'objet d'un numéro spécifique récemment paru dans Émulations ${ }^{3}$.

\section{La santé au prisme des rapports sociaux}

Parler d'inégalités, c'est considérer que les différenciations sociales observées dans le champ de la santé renvoient à un gradient social de santé qui traverse, de part en part, l'ensemble du corps social. Cette perspective se distingue des approches en termes de " santé-précarité » qui s'intéressent à la santé des plus démunis et s'accompagnent généralement d'actions visant " une population, aux contours flous, qualifiée de précaire, exclue ou marginalisée », dont l'état de santé serait dégradé comparativement au reste de la population (Haschar-Noé, Lang, 2018 : 16). Ce dossier considère plutôt que comprendre les inégalités suppose de s'intéresser à l'ensemble du corps social. Les contributions de Déborah Ridel et de Leslie Fonquerne usent ainsi de la comparaison et montrent bien l'impact de l'appartenance sociale des patients dans leurs prises en charge et leurs parcours de soins. Déborah Ridel détaille des disparités dans l'accueil aux urgences des patients en fonction des propriétés sociales que leur impute le personnel soignant, ce qui lui permet de mettre en lumière l'existence de traitements différenciés fondés sur des critères tels que l'hexis corporelle, le langage ou encore la littératie en santé. Leslie Fonquerne s'intéresse quant à elle aux parcours contraceptifs en fonction du profil sociologique des patientes. Elle montre que toutes les femmes ne bénéficient pas d'un choix contraceptif libre et éclairé, mais que la manière dont les femmes sont conduites à « choisir » leur mode de contraception est empreinte d'inégalités. Les femmes les moins favorisées ainsi que celles perçues comme « déviantes » par rapport à la norme médicale de l'observance sont, par exemple, davantage susceptibles de se voir prescrire des contraceptifs de long cours comparativement aux usagères les plus dotées en capitaux.

\footnotetext{
${ }^{3}$ Numéro 27 (2018) : «Les maladies chroniques dans les Suds. Expériences, savoirs et politiques
} aux marges de la santé globale ». 
La notion de discrimination invite en outre à resituer les différences de traitement fondées sur des critères prohibés par la loi comme « les rouages de la (re)production des rapports de pouvoir structurels qui engendrent des groupes sociaux majoritaires et minoritaires » (Bereni, Chappe, $2011: 16)$. Comme dans le domaine de l'emploi, les discriminations en santé sont particulièrement interrogées au prisme du critère ethnoracial (Carde, 2007; Cognet, Hamel, Moisy, 2012 ; Coutant, 2010 ; Toulze, Meidani, 2017). Ces travaux participent, en cela, à renouveler la recherche sur les inégalités sociales de santé qui, dans le monde francophone, a longtemps été peu attentive aux effets des rapports sociaux de racisation (Fassin, 2008). Bien qu'initialement affiliée à la question du racisme et essentiellement attentive au sort des descendants d'immigrés, la notion de discrimination renvoie aujourd'hui à un ensemble de critères parmi lesquels ressortent tout particulièrement ceux du sexe, de l'origine, de l'âge, du handicap et de l'orientation sexuelle. Penser les discriminations amène ainsi à saisir les inégalités dans leurs dimensions plurielles et invite à s'intéresser à l'articulation des rapports sociaux, comme le font les travaux adossés à une perspective intersectionnelle.

Ce numéro atteste bien en effet que s'il existe un lien entre l'état de santé d'une personne et sa position dans la hiérarchie sociale, cette position n'est pas uniquement définie par son appartenance de classe, mais aussi par son genre, son âge et son origine réelle ou supposée. Les différentes contributions s'intéressent à la manière dont les rapports sociaux de classe, de sexe ou de « race » qui structurent l'espace social impactent les corps. Elles portent à la fois sur les effets de l'origine - réelle ou supposée - sur la santé (R. Braud ; L. Virole,), sur ceux des normes de genre et du sexisme (L. Fonquerne ; C. Pezeril), ainsi que du statut socioéconomique ou de la position dans la hiérarchie du travail (E. Carde ; C. Desprès, P. Lombrail ; D. Ridel). Les interactions, les articulations entre ces différents rapports sociaux ainsi que leurs effets combinés sur la santé sont d'ailleurs au centre de plusieurs articles (R. Braud ; S. Morel, V. Cottereau ; P. Perrenoud ; C. Pezeril ; L. Virole). Cette perspective systémique permet de prendre en considération, dans une approche intersectionnelle, l'articulation des rapports sociaux de classe, de sexe et de "race » et leurs effets dans la production des inégalités sociales de santé (Bilge, 2010 ; Kergoat, 2009).

\section{Expériences des patient·e·s / pratiques des professionnel·le·s}

Ce numéro a également pour originalité d'éclairer le processus de constitution des inégalités en s'appuyant tout autant sur le vécu des patient·e·s que sur celui des professionnel-le·s, plus rarement abordé pour éclairer cet objet.

Les articles s'intéressent ainsi à l'accès aux soins des patient·es et, plus largement, à leurs prises en charge et à leurs parcours de soins à partir de leurs expériences. En s'appuyant sur le vécu de malades perçus comme "immigrés » et atteints de diabète, Rosane Braud montre de quelle manière l'adaptation des prises en charge, encouragées par les orientations politiques actuelles, peut avoir des effets délétères sur leur santé. Les entretiens révèlent que les soins sont adaptés à des pratiques socioculturelles plus 
souvent supposées que réelles, alors que ce sont la plupart du temps des contraintes ordinaires et économiques qui font obstacle à la bonne adoption des comportements alimentaires recommandés. De la même manière, Louise Virole s'intéresse à la qualité des soins telle qu'elle est perçue par les usagères en interrogeant des femmes étrangères enceintes sur l'accompagnement dont elles bénéficient. S’il apparaît que les usagères sont satisfaites de leurs prises en charge dans les dispositifs existants, l'auteure montre qu'il faut cependant relativiser leur satisfaction, car leurs attentes sont façonnées par le suivi obstétrique qu'elles ont éprouvé dans leurs pays d'origine. Ce travail montre finalement que tous les usagers du système de soins n'ont pas des attentes équivalentes, mais que leurs positions de classe, de genre et d'origine modulent leur niveau d'exigence. Sylvie Morel et Victoire Cottereau se penchent quant à elles sur l'expérience de migrants " primo-arrivants » atteints du VIH. Pour les personnes ayant appris leur séropositivité avant leur arrivée en France, les discriminations directes vécues dans leur pays d'origine sont fréquemment reportées et peuvent jouer un rôle central dans leur parcours de migration. Les " primo-arrivants » doivent également surmonter de nombreux obstacles pour avoir accès aux traitements, l'ouverture des droits impliquant notamment des démarches administratives complexes pour lesquelles des barrières légales et des pratiques discriminatoires sont observées. Enfin, le travail de Charlotte Pezeril souligne à quel point le vécu de la discrimination ne saurait se réduire à une atteinte émotionnelle, en ce qu'elle entraîne bien souvent des conséquences particulièrement défavorables pour l'état de santé, tout comme elle peut être à l'origine de nombreuses ruptures dans la trajectoire des victimes.

De manière complémentaire, d'autres contributions s'intéressent aux professionnel-le·s de santé et mettent l'accent sur leur propre vécu et la manière dont ils expliquent leurs pratiques. Estelle Carde explore le sentiment de difficulté qu'éprouvent des médecins généralistes face à leurs patients socialement défavorisés. Elle s'attache à restituer les principales sources des difficultés éprouvées dans le suivi de ces patients. Patricia Perrenoud s'intéresse aux difficultés que rencontrent les sages-femmes lorsqu'elles suivent des femmes « en situation de détresse sociale » durant la période périnatale. Caroline Desprès et Pierre Lombrail explorent de leur côté les logiques sociales des refus de soins à l'égard des bénéficiaires de la CMU complémentaire par des professionnel-le·s de santé de ville. À partir d'une analyse socioanthropologique de leurs discours, ils s'intéressent à la fois aux logiques d'acteurs (matérielles et symboliques), à l'impact de l'environnement sociétal (sociopolitique), institutionnel (organisation des soins, politiques de santé, normes professionnelles et administratives) et économique. Enfin, le travail de Déborah Ridel montre l'impact des contraintes structurelles, qui enjoignent les soignant·e.s à agir et décider de plus en plus vite dans la prise en charge des patient·e.s. On comprend ainsi combien il est nécessaire de tenir compte des conditions concrètes d'activité pour mieux comprendre les manières de faire et de penser des soignant·e.s. 
Ces contributions permettent de restituer les vécus, ainsi que les représentations, les catégorisations et les pratiques de triage observées et/ou décrites par les professionnel-le·s dans un contexte plus large, façonné par des logiques managériales, des contraintes organisationnelles et une organisation du travail qui en composent le terreau. L'étude des expériences des patient·e·s et celle des pratiques des professionnel-le·s se répondent l'une et l'autre, alimentant la réflexion sur les inégalités sociales de santé et mettant au jour les paradoxes des actions qui visent à les combattre.

\section{Les paradoxes de la lutte contre les inégalités et les discriminations en santé}

Ce numéro aborde aussi la question de la lutte contre les inégalités et les discriminations en santé. Il permet de pointer la lutte silencieuse que mènent certains professionnel-le·s de santé afin de préserver l'éthique soignante et/ou humanitaire, cet aspect étant très rarement investigué. Sylvie Morel et Victoire Cottereau, qui observent que les professionnel-le.s hospitaliers travaillent dans un contexte de plus en plus contraint par des politiques migratoires et gestionnaires qui durcissent l'accès aux droits, entravent l'accès aux traitements et la continuité des soins, décrivent ainsi des " pratiques buissonnières » qui prennent la forme de «bricolages", parfois aux limites de la légalité, visant à permettre l'accès aux soins et la poursuite des traitements aux patient·e.s. Estelle Carde, de la même manière, montre bien que face à leurs patient·e.s défavorisé·ess, les médecins adaptent leur pratique médicale, par exemple en s'astreignant à des consultations plus longues et plus fréquentes ou en les accompagnant dans leurs prises de rendez-vous avec d'autres professionnel.le.s. Patricia Perrenoud décrit quant à elle l'engagement des sages-femmes indépendantes en Suisse romande et leur action auprès de familles marquées par des situations de crise durant la période postnatale. Elle rend toutefois compte des difficultés, pour ces praticiennes, d'obtenir de l'aide auprès de leur réseau professionnel, notamment des médecins et des sagesfemmes exerçant à l'hôpital.

Les différentes contributions révèlent également en contrepoint les difficultés et les effets ambivalents des politiques et dispositifs visant à lutter contre les inégalités en santé. Louise Virole interroge ainsi les effets des prises en charge dédiées sur les parcours de soins de femmes enceintes primo-arrivantes. Elle montre que si les dispositifs mis en place améliorent l'accès aux soins de ces femmes, le caractère marginal et opaque des dispositifs, souvent dérogatoires du droit commun, limite leur action. En contournant les barrières à l'accès aux soins, ces dispositifs creusent l'écart entre les usagères prises en charge au sein des dispositifs dédiés et celles qui en sont exclues. Dès lors, ils ne permettent pas de lutter de manière globale contre les inégalités de santé. De la même manière, le travail de Rosane Braud montre bien que les orientations politiques actuelles, qui incitent à cibler des publics dits "précaires » et « immigrés ", conduisent en réalité à une distribution différenciée des soins qui participe le plus souvent à reproduire des inégalités sociales. 
Les auteur.e.s des contributions soulignent enfin un dernier paradoxe de la lutte contre les inégalités et les discriminations en santé : si l'intersectionnalité constitue un cadre d'analyse utile pour décrire l'expérience des minorités, les professionnel-le·s tout comme les institutions ont pourtant des difficultés à appréhender les croisements et les articulations des rapports de domination. Charlotte Pezeril montre ainsi que l'institution belge habilitée à recevoir les plaintes pour discrimination liée au VIH possède un fonctionnement qui tend à invisibiliser leur caractère intersectionnel. Il apparaît en effet qu'au niveau institutionnel, légal et judiciaire, rien n'est fait pour prendre en compte et traiter les discriminations multiples, ce qui tend à la fois à les dépolitiser et à masquer les rapports de force, le contexte et l'historicité dans lesquels elles s'inscrivent.

Pierre Aïach remarquait, dès le milieu des années 1970, que les inégalités dans le domaine de la santé sont rarement "ressenties et dénoncées comme une injustice ", tant elles semblent naturelles et inscrites dans l'ordre de choses (Aïach, 1978 : 465). Aujourd'hui encore, les représentations ordinaires placent souvent le corps et son bon fonctionnement du côté de la nature, oubliant combien il reste soumis aux rapports de domination qui structurent nos sociétés. Ce numéro vient nous rappeler à quel point nous sommes inégaux en matière de santé. Nos corps sont façonnés par nos contraintes ordinaires. Nos parcours de soins et expériences de santé restent quant à eux étroitement liés à nos propriétés sociales.

Ce numéro s'inscrit ainsi dans le prolongement d'un ensemble de travaux visant à mieux comprendre les inégalités sociales de santé et invite à renouveler et à approfondir les questionnements sur cette thématique, l'action contre les inégalités sociales de santé et la lutte contre les discriminations apparaissant également de manière transversale. Les pistes de recherche futures demeurent nombreuses et doivent nous permettre d'avancer vers une compréhension toujours plus fine des mécanismes à l'œuvre dans la production des inégalités. Cette connaissance est indispensable à la mise en place de politiques de réduction efficaces des inégalités, mais aussi - comme le propose la conclusion de ce numéro - pour déjouer l'invisibilisation des discriminations. Puissent donc ces articles être la base d'émulations tant scientifiques que politiques.

\section{Bibliographie}

AïAch P. (1978), « Point de vue sur la sociologie de la morbidité », Santé, médecine et société, Paris, Éditions CNRS INSERM.

AÏach P., Niewiadomski C. (2008), Lutter contre les ISS, Politiques publiques et pratiques professionnelles, Rennes, Presses de l’EHESP (« Recherche Santé Social »). 
Arber S., Meadows R. (2020), "Class inequalities in health and the Coronavirus: a cruel irony? ", The blog of the Department of Sociology at the University of Surrey, Department of Sociology, Surrey University. En ligne. URL : https://blogs.surrey. ac.uk/sociology/2020/03/23/class-inequalities-in-health-and-the-coronavirus-acruel-irony/.

Bajos N., Warszawsiki J., Pailhé A., Counil E., Jusot F., Spire A., Martin C., Meyer L., Sireyjol A., Frank J.-E., Lydie N. (2020), " Les inégalités sociales au temps du COVID-19 », Questions de santé publique, $\mathrm{n}^{\circ} 40$.

Balard F., Corvol A. (2020), " Covid et personnes âgées : liaisons dangereuses ", Gérontologie et société, vol. 42, n 162, p. 9-16.

BERENI L., CHAPPE V.A. (2011), « La discrimination, de la qualification juridique à l'outil sociologique », Politix, $\mathrm{n}^{\circ}$ 94, p. 7-34.

Bessière C., Biland E., Gollac S., Marichalar P., Minoc J. (2020), « Penser la famille au temps du Covid-19 », Mouvements. En ligne. URL : https://mouvements.info/penser-lafamille-aux-temps-du-covid-19/.

BILGE S. (2010), « De l'analogie à l'articulation : théoriser la différenciation sociale et l'inégalité complexe », L’Homme et la société, vol.176-177, n² 2, p. 70-88.

Bihr A., Pfefferkorn R. (2008), Le système des inégalités, Paris, La Découverte.

Bourdieu P. (1997), Méditations pascaliennes, Paris, Seuil.

Bourdieu P. (1979), La distinction, Paris, Éditions de Minuit.

Brun S., Simon P. (2020), « L'invisibilité des minorités dans les chiffres du Coronavirus : le détour par la Seine-Saint-Denis », De facto. En ligne. URL : http://icmigrations. $\mathrm{fr} / 2020 / 05 / 15 /$ defacto-019-05/.

CARDE E. (2020), « La Covid-19 creuse les inégalités d'aujourd'hui, mais aussi celles de demain ", The Conversation. En ligne. URL : https://theconversation.com/la-covid-19creuse-les-inegalites-daujourdhui-mais-aussi-celles-de-demain-138288.

CARDE E. (2007), "Les discriminations selon l'origine dans l'accès aux soins », Santé Publique, vol. 19, n² 2, p. 99-109.

Chung R.Y.-N., Li M.M. (2020), « Anti-Chinese sentiment during the 2019-nCoV outbreak», The Lancet, $n^{\circ} 395$, p. 686-687.

Cockerham W.C. (2016), The New Blackwell Companion to Medical Sociology, Oxford, Wiley-Blackwell.

Cognet M., Hamel C., Moisy M. (2012), « Santé des migrants en France : l'effet des discriminations liées à l'origine et au sexe », Revue européenne des migrations internationales, vol. 28, p. 11-34.

Contretemps (2020), « Dossier : Inégalités renforcées, précarité amplifiée. Sous la pandémie le patriarcat », Contretemps. En ligne. URL : https://www.contretemps.eu/ dossier-patriarcat-covid19/. 
Counil E., Khlat M. (2020), « Covid-19: is there a disproportionate burden on working classes in France? », The Conversation. En ligne. URL : https://theconversation.com/ covid-19-is-there-a-disproportionate-burden-on-working-classes-in-france-137533.

CoUTANT I., 2010, « L'origine est-elle discriminante dans la prise en charge de la déviance? Une étude dans un service de psychiatrie pour adolescents », in D. FASsin (dir.), Les nouvelles frontières de la société française, Paris, La Découverte, p. 291-313.

Devakumar D., Shannon G., Bhopal S. S., Abubakar I. (2020), « Racism and discrimination in COVID-19 responses », The Lancet, vol. 395, n 10231, p. 1194.

Dodier N., Camus A. (1997), "L'admission des malades. Histoire et pragmatique de l'accueil à l'hôpital », Annales. Histoire, Sciences Sociales, 52 année, n 4, p. 733-763.

DREES (2017), L'état de santé de la population en France - Rapport 2017, Paris, Santé publique France/DREES.

Dubet F. (2011), "Régimes d'inégalité et injustices sociales », SociologieS, Rubrique « Débats : Penser les inégalités ». En ligne. URL : http://journals.openedition.org/ sociologies $/ 3643$.

Dubost C.L., Pollak C., Rey S. (2020), « Les inégalités sociales face à l'épidémie de Covid19 ", Dossiers de la DREES, États des lieux et perspectives, $n^{\circ} 62$, juillet.

Duvoux, N. (2017), Les inégalités sociales, Paris, PUF (« Que sais-je ?»).

Fassin D. (2000), «Qualifier les inégalités », in A. Leclerc, D. Fassin, H. Granjean, M. Kaminski, T. LANG (dir.), Les inégalités sociales de santé, Paris, La Découverte, p. 123-144.

Fassin D. (2008), « Les impensés des inégalités sociales de santé », in C. NiewiadomsKi, P. AïACH (dir.), Lutter contre les inégalités sociales de santé. Politiques publiques et pratiques professionnelles, Rennes, Presses de l'EHESP, p. 21-28.

GeLLy, M. (2016), « Des inégalités en tous genres face au décès par sida et de leur ignorance par le système de santé », Agone, $\mathrm{n}^{\circ}$ 58, p. 35-150.

Gelly M., PitTi L. (2016), « Une médecine de classe ? Inégalités sociales, système de santé et pratiques de soins », Agone, $\mathrm{n}^{\circ}$ 58, p. 7-18.

Glaser B.G., Strauss A. (1967), The Discovery of Grounded Theory: Strategies for Qualitative Research, Chicago, Aldine Publishing Company.

Haschar-Nó́ N., LANg T. (2018), Réduire les inégalités sociales de santé, Toulouse, PUM (« Sciences sociales - Santé Société »).

Haut Comité de Santé Publique (2009), Les inégalités sociales de santé. Sortir de la fatalité, Paris, HCSP.

Hunt K., Macintyre S. (2000), "Genre et inégalités sociales en santé », in A. Leclerc, D. Fassin, H. Grandjean, M. Kaminski, T. Lang (dir.), Les inégalités sociales de santé, Paris, La Découverte (« Recherches »), p. 363-375.

KeRgOat D. (2009), « Dynamique et consubstantialité des rapports sociaux », in E. DorLIN (dir.), Sexe, race, classe, pour une épistémologie de la domination, Paris, PUF, p. 111-125. 
Kivits J., BaLARD F., FouRnier C., WinAnCE M. (dir.) (2016), Les recherches qualitatives en santé, Malakoff, Armand Colin.

Lahire B. (2019), Enfances de classe. De l’inégalité parmi les enfants, Paris, Seuil.

Lambert A., Cayouette-Remblière J., Guéraut E., Bonvalet C., Girard V., Le Roux G., Langlois L. (2020), « Logement, travail, voisinage et conditions de vie : ce que le confinement a changé pour les Français », COronavirus et CONfinement : Enquête longitudinale, Note de synthèse $n^{\circ} 10$, vague 6 , INED.

LANG, T. (2018), "Si nous considérions la réduction des inégalités sociales de santé comme étant l'affaire de tous ?», Santé Publique, s1(HS1), p. 7-9.

Leclerc A., Fassin D., Grandjean H., Kaminski M., Lang T. (2000), Les inégalités sociales de santé, Paris, La Découverte ( Recherches »).

Mackenbach J. P., Stirbu I., Roskam A.-J., Schaap M. M., Menvielle G., Leinsalu M., Kunst A. E. (2008), " Socioeconomic Inequalities in Health in 22 European Countries », New England Journal of Medicine, vol. 23, $n^{\circ}$ 358, p. 2468-2481.

Mackenbach J.P., Bakker M. (2002), Reducing Inequalities in Health: A European Perspective, London, Routledge.

Marmot M. (2020), « Society and the slow burn of inequality », The Lancet, $n^{\circ} 395, p$. 1413-1414.

Marmot M., Friel S., Bell R., Houweling T.A.J., Taylor S. (2008), « Closing the gap in a generation: Health equity through action on the social determinants of health », The Lancet, vol. 9650, $\mathrm{n}^{\circ}$ 372, p. 1661-1669.

Membrado M. (2006), " Les femmes dans le champ de la santé : de l'oubli à la particularisation », Nouvelles Questions Féministes, vol. 25, p. 16-31.

Menvielle G., Stirbu I., Roskam A.J., SchaAp M., Leinsalu M., Kunst A., Mackenbach J. (2009), «Les inégalités sociales de mortalité en Europe », Medecine/Sciences, n² 25, p. $192-196$.

Noppert G.A. (2020), «États-Unis : pourquoi les Noirs et les pauvres sont les plus touchés par le Covid-19», The Conversation. En ligne. URL : https://theconversation. com/etats-unis-pourquoi-les-noirs-et-les-pauvres-sont-les-plus-touches-par-lecovid-19-136538.

Noûs C. (2020), « Le covid-19, la guerre et les quartiers populaires », La Nouvelle Revue du travail. En ligne. URL : http://journals.openedition.org/nrt/6771.

Papon S., Robert-Bobée I. (2020), « Une hausse des décès deux fois plus forte pour les personnes nées à l'étranger que pour celles nées en France en mars-avril 2020 », Insee Focus, $\mathrm{n}^{\circ} 198$, juillet.

Passeron J.C. (1981), " Le sens et la domination. Différences dans la différence », in F. Chevaldonné (dir.), La Communication inégale. L'accès aux médias dans les campagnes algériennes, Paris, CNRS, p. 7-14. 
PeRRot P. (1991), Le corps féminin. Le travail des apparences XVIII-XIX'e siècle, Paris, Seuil (« Points »).

Poiret C. (2011), « Les processus d'ethnicisation et de raci(ali)sation dans la France contemporaine : Africains, Ultramarins et "Noirs" ", Revue européenne des migrations internationales, vol. 27, $\mathrm{n}^{\circ}$ 1, p. 107-127.

E. Recchi et al. (2020), « Confinement pour tous, épreuve pour certains. Les résultats de la première vague d'enquête du projet CoCo », Policy Brief, $\mathrm{n}^{\circ} 1$.

Renard S., Rebourg M. (2020), « De l'éventualité d'une prolongation du confinement spécifique aux personnes âgées : que sommes-nous prêts à sacrifier ? », Revue des droits et libértés fondamentaux, $\mathrm{n}^{\circ}$ 30. En ligne. URL : http://www.revuedlf.com/droitadministratif/de-leventualite-dune-prolongation-du-confinement-specifique-auxpersonnes-agees-que-sommes-nous-prets-a-sacrifier/.

SCAMbler G. (2020), " Covid-19 as a "breaching experiment": exposing the fractured society », Health Sociology Review, vol. 2, n² 29, p. 140-148.

Scodellaro C., Pan Ké Shon J., Legleye S. (2017), « Troubles dans les rapports sociaux : le cas de l'anorexie et de la boulimie », Revue française de sociologie, vol. 58, p. 7-40.

Toulze M., Meidani M. (2017), «Santé et discriminations », Les cahiers de la LCD, n 5 , Paris, L'Harmattan.

Van Oyen H., Charafeddine R., Deboosere P. et al. (2011), " Contribution of mortality and disability to the secular trend in health inequality at the turn of century in Belgium », European Journal of public Health, vol. 21, n 6, p. 781-787.

Vidal C., Salle M. (2017), Femmes et santé, encore une affaire d'hommes ?, Paris, Belin («Égale à égal »).

WilLIAMSON E. et al. (2020), « OpenSAFELY: factors associated with COVID-19-related hospital death in the linked electronic health records of 17 million adult NHS patients », medRxiv preprint. 